\title{
El papel de la epidemiología en la investigación de los trastornos mentales
}

\author{
Guilherme Borges, Dr en C, ${ }^{(1,2)}$ María Elena Medina-Mora, Dra en Psicol Soc, ${ }^{(2)}$ Sergio López-Moreno, Dr en C. ${ }^{(2,3)}$
}

\section{Borges G, Medina-Mora ME, López-Moreno S. El papel de la epidemiología en la investigación de los trastornos mentales Salud Publica Mex 2004;46:451-463.}

\section{Resumen}

Los trastornos mentales, incluyendo los trastornos del uso de sustancias, hacen ya parte del panorama epidemiológico de México y seguirán en el escenario nacional por las próximas décadas, incrementando incluso su presencia como causa de enfermedad, discapacidad y muerte en nuestro país. Por lo tanto, el manejo epidemioló gico de estos problemas se hace urgente. Este trabajo busca plantear el campo de estudio de la epidemiología de los trastornos mentales y sus limitaciones, haciendo énfasis en los elementos comunes de ésta con otras áreas más tradicionales de la epidemiología y en las aportaciones particulares de este campo epidemio lógico a la psiquiatría en general y a la epidemiologia en particular. Planteamos a continuación los diseños y problemas más comunes en este campo de la epidemiología, su utilidad para acciones de prevención, y señalamos los retos que nos esperan en el futuro. Una característica distintiva de esta área es que los trasto rnos mentales se manifiestan en dos niveles, como conducta (por ejemplo, una conducta compulsiva de lavarse las manos) y como elemento de la vida mental del sujeto (por ejemplo, el pensamiento obsesivo sobre las bacterias que se encuentran presentes en todos lados y que son una fuente constante de amenaza para algunos sujetos). Debido a esto, mucho de lo que sabemos sobre estos fenómenos proviene del autorreporte que el sujeto es capaz de hacer sobre sus sentimientos (introspección), ya sea en una conversación con un clínico entrenado 0 expresándolos al endosar reactivos en un cuestionario estandarizado. 0 tro aspecto importante a resaltar

\author{
Borges G, Medina-Mora ME, López-Moreno S. \\ The role of epidemiology \\ in mental disorder research. \\ Salud Publica Mex 2004;46:451-463.
}

(1) Instituto $\mathrm{N}$ acional de Psiquiatría Ramón de la Fuente Muñiz, Secretaría de Salud. México, DF, México.

(2) División de Ciencias Biológicas y de la Salud, Universidad Autónoma Metropolitana, Unidad Xochimilco. México, DF, México.

(3) Instituto N acional de Salud Pública. Cuernavaca, Morelos, México.

Solicitud de sobretiros: Dr.G uilherme Borges. Departamento de Investigaciones Epidemiológicas, Dirección de Investigaciones Epidemiológicas y Psicosociales, Instituto N acional de Psiquiatría Ramón de la Fuente Muñiz. Calzada México-X ochimilco 101, colonia San Lorenzo Huipulco, 14370 México, DF, México. Correo electrónico: guibor@imp.edu.mx 
es la necesidad de hablar en plural al referirnos a los trastornos mentales. Este campo de la epidemiología presenta también un carácter particular, bifacético: por un lado, este es un problema en sí mismo, que produce sufrimiento y motiva la búsqueda de atención especializada, con manifestaciones clínicas particulares. Por otro lado, esta epidemiología también se orienta hacia un dominio particular de determinantes (como el uso, abuso o dependencia hacia las drogas) y cómo estas variables independientes afectan determinados procesos y enfermedades (como los accidentes, el homicidio, el suicidio, la cirrosis hepática, etc.) Por último, la epidemiología de los trastornos mentales se ha caracterizado también por su interés en una serie de procesos que no parecen constituir síndromes pro piamente dichos, pero que son a todas luces de interés sanitario, siendo el ejemplo más claro de esto el problema de la violencia. La epidemiolo gía de los trastornos mentales se enfrenta a enormes retos en el nuevo milenio. D ebe hacer frente a un panorama epidemiológico complejo y cambiante. Los aspectos más importantes de su desarrollo futuro se vinculan con los siguientes puntos: medición de los trastornos mentales y de los factores de riesgo, el diseño y métodos de muestreo más eficientes, la relación entre la investigación bioló gica, la genética, las disciplinas sociales y la epidemiología, y la interfase entre la epidemiología y la evaluación de los tratamientos y los servicios.

Palabras clave: epidemiología psiquiátrica; trastornos mentales; estudios poblacionales; factor de riesgo; México tors (drug use, abuse, or addiction) and on the way these independent variables result in certain processes and outcomes (such as accidents, homicide, suicide, liver cirrhosis, etc.). Finally, the epidemiology of mental disorders has also been set apart by its focus in series of processes that are not suitably classified as syndromes, but which are germane to public health, for example, violence.The epidemiolo gy of mental disorders faces great challenges in the new millennium, including a complex, changing epidemiolo gic scenario. Several important issues will influence the future development of mental disorder epidemiology: measurement of mental disorders and risk factors, more efficient sampling design and methods, the relationships among biological research, genetics, social studies, and epidemiology, and the interface between epidemiology and the evaluation of therapies and health services.

Key words: psychiatric epidemiology; mental disorders; population-based studies; risk factor; Mexico

\section{¿Por qué una epidemiología de las enfermedades mentales?}

A mediados de los años noventa, la Universidad de Harvard y la Organización Mundial de la Salud (OMS) publicaron, en el ahora famoso texto The Global Burden of Disease, ${ }^{1}$ una noticia que sorprendió a muchos: para el año 2020, la depresión mayor unipolar sería la segunda causa de enfermedad más importante en el mundo, en términos de su peso global. A esta conclusión se llega al evaluar la enfermedad mediante indicadores compuestos -como el de años de vida saludable ajustados por discapacidad o por muerte prematura (AVISA) - y no únicamente por el volumen de su mortalidad. Cuando se utilizan indicadores compuestos, los trastornos mentales que tienen una larga duración, una edad de inicio más temprana, naturaleza crónica, alta prevalencia, pocos tratamientos eficaces y alto riesgo de producir discapacidad, incrementan su importancia como problemas de salud pública. ${ }^{2}$ Además de la depresión mayor unipolar, en este gru- po de padecimientos se encuentran los desórdenes bipolares, el suicidio, los trastornos obsesivo-compulsivos, la esquizofrenia y los trastornos derivados del uso de alcohol, los que se convierten en un rubro de primera importancia cuando se considera la pérdida de AVISA que provocan. ${ }^{3}$ Por esta razón, el estudio de los trastornos mentales es una parte sustantiva de la investigación epidemiológica y seguirá siéndolo mientras su presencia como causa de enfermedad y discapacidad sea tan importante.

En este trabajo nos proponemos demostrar que la investigación epidemiológica de los trastornos mentales se encuentra en un claro proceso de especialización, y que éste resulta de la especificidad epidemiológica de dichos trastornos, la cual hace que sea muy difícil, si no imposible, usar los criterios tradicionalmente aplicados al estudio de otros problemas de salud (como, por ejemplo, las infecciones y las enfermedades crónico-degenerativas). Igualmente, la especificidad clínica de los trastornos mentales ha obligado a los epidemiólogos a utilizar modalidades muy propias 
para definir lo que es un caso, un control, un expuesto, y un no expuesto, por ejemplo. Al final, estas especificidades han terminado por conformar un espacio que difiere de otros campos de la investigación epidemiológica en forma aparentemente sutil, pero muy importante. Al final se señalan algunas de las ventajas, limitaciones y retos que esta especificidad impone a la investigación epidemiológica contemporánea.

\section{Epidemiología de los trastornos mentales: campo de estudio y limitaciones}

La epidemiología de los trastornos mentales -o epidemiología psiquiátrica- es el conjunto de saberes que, utilizando los principios, conceptos, métodos y estrategias de investigación de la epidemiología, se encarga del estudio de la salud mental. Como han señalado Anthony, Eaton y Henderson, ${ }^{4}$ una característica distintiva de esta área es que los trastornos mentales se manifiestan en dos niveles: a) como conducta (por ejemplo, la conducta compulsiva de lavarse las manos) $y, b$ ) como elemento de la vida mental del sujeto (por ejemplo, el pensamiento obsesivo sobre las bacterias que se encuentran presentes en todos lados y que son una fuente constante de amenaza para algunos sujetos). Esta complejidad se manifiesta a veces en la necesidad de considerar diferentes aspectos de los que parecería ser, en principio, una misma epidemiología. Por ejemplo, en el campo de la investigación epidemiológica del suicidio podemos distinguir en primera instancia el estudio del suicidio consumado. Este es un evento (violencia auto-infligida) que tiene problemas de medición particulares, en la medida en que debemos inferir que el acto lo realizó la propia persona, y debemos juzgar que la persona actuó con la intención de matarse. Pero además del suicido consumado, este campo incluye también los intentos de suicidio (que son conductas en principio observables externamente), los planes (que sólo parcialmente pueden ser observables, pues muchas veces sólo existen en la mente del sujeto) y la ideación reiterativa y persistente sobre la muerte de uno mismo, es decir, la ideación suicida que, como lo indica su nombre, existe principalmente como parte de la vida mental del sujeto. ${ }^{5}$

El hecho de que una parte importante de los problemas a los que se dedica la epidemiología psiquiátrica tenga que ver con la vida mental del individuo le plantea un aspecto distintivo: mucho de lo que sabemos sobre estos fenómenos proviene del autorreporte que el sujeto es capaz de hacer sobre sus sentimientos, ya sea en una conversación con un clínico entrenado o al contestar reactivos con un cuestionario estandarizado. A esto se le puede añadir la observación de con- ductas (especialmente en los trastornos psicóticos) y la evaluación del paciente realizando tareas cognitivas (especialmente en los trastornos demenciales). ${ }^{6}$

Aunque se han realizado muchos progresos en el conocimiento de las funciones del sistema nervioso central y de sus estructuras, hasta el momento no existe ningún marcador objetivo de trastorno mental, ni siquiera en áreas particulares de estudio, como en la epidemiología de los trastornos por uso de sustancias psicotóxicas. ${ }^{7}$ Así, una parte sustancial de la epidemiología psiquiátrica se ha dedicado a la elaboración de instrumentos que tienen como base el autorreporte del entrevistado para la medición de trastornos mentales en comunidad, como se discute más adelante. ${ }^{8}$

Otro aspecto importante es la necesidad de hablar en plural al referimos a los trastornos mentales. La epidemiología psiquiátrica actual ha favorecido la noción de que es poco provechoso hablar de enfermedad mental en general, de la misma manera que no se plantea una epidemiología de la enfermedad crónica o de la enfermedad transmisible, en singular. La clasificación de los trastornos mentales combina la tradición norteamericana -mediante el Diagnostic and Statistical Manual of Mental Disorders, $4^{\text {a }}$ Revisión, ${ }^{9}$ también llamado DSM-IV- con la tradición internacional -con la Clasificación Estadística Internacional de Enfermedades y Problemas Relacionados con la Salud, $10^{\text {a }}$ revisión $^{10}$ (CIE-10). Ambas clasificaciones distinguen los trastornos mentales con base en su sintomatología. Como resultado, la epidemiología psiquiátrica abandonó la postura que planteaba que era imposible realizar diagnósticos específicos de trastornos mentales en estudios comunitarios, y que sólo aspiraba a catalogar a los individuos en un continuo de salud-enfermedad mental, que algunos llamaron "tensión psicológica", "distress" o "desmoralización".6,11,12 La investigación actual ha demostrado que existe una gran variabilidad y posibilidad de precisión diagnóstica en los trastornos de la emoción, la cognición y la conducta ${ }^{13}$ así como en sus factores de riesgo, evolución y pronóstico.

Aun dentro de esta caracterización general de la epidemiología psiquiátrica debe resaltarse el hecho de que habitualmente este campo presenta dos facetas. ${ }^{14}$ Tomemos como ejemplo a la epidemiología de la farmacodependencia: en sí mismo, este problema produce sufrimiento y manifestaciones clínicas que motivan la búsqueda de atención especializada. Existe aquí una epidemiología centrada en los cuadros de dependencia y abuso, ${ }^{15} \mathrm{o}$ en formas de consumo que se consideran por sí mismas un daño a la salud (por ejemplo, el alcoholismo consuetudinario o el abuso del tabaco). ${ }^{16,17}$ Desde esta perspectiva, la variable dependiente (la 
dependencia al alcohol o al tabaco, por ejemplo) es el interés primario. Pero, por otro lado, la epidemiología de las adicciones puede estudiar la forma como el patrón de consumo de cierta sustancia (por ejemplo, el alcohol) determina el desarrollo de otros procesos y enfermedades (como los accidentes, el homicidio, el suicidio). Aquí, el interés primario es determinar el efecto que tiene la exposición al psicofármaco sobre alguna condición de salud, contingente a la presencia de otros factores. Otros campos especializados de la epidemiología de los trastornos mentales comparten esta característica de presentar una doble faceta, en mayor o menor medida. Por ejemplo, es posible estudiar la depresión como variable dependiente, investigando los factores que se asocian a ella como condiciones de riesgo, o podemos estar interesados en la depresión como el factor determinante de otros daños (como el suicidio consumado). En este sentido se comprende claramente que ciertas áreas de interés reciente, como ha sucedido con los aspectos sociales y económicos relacionados con los trastornos mentales, puedan abordarse como factores de riesgo o como consecuencia de dichos trastornos. Este es el caso del desempeño de ciertos roles ocupacionales, la deserción escolar, el fracaso matrimonial y el embarazo temprano, por ejemplo. . $^{18,19}$

Ya sea en una $u$ otra de estas facetas, los problemas básicos a los que se dedica esta epidemiología son comunes a cualquier otra epidemiología, a saber: la determinación de la frecuencia del padecimiento (¿cuántos?), su distribución (¿en dónde?), los grupos más afectados (¿quiénes?), los procesos que determinan el padecimiento (¿cómo?) y las acciones de intervención y prevención (¿qué hacer?). ${ }^{20}$

Finalmente, la epidemiología de los trastornos mentales se ha caracterizado también por su interés en una serie de procesos que no parecen constituir síndromes propiamente dichos, pero que a todas luces son de interés sanitario. El ejemplo más claro de esto es el problema de la violencia. Desde este rubro podemos pensar en fenómenos tan amplios como la guerra, y tan específicos como una riña en un bar. Es claro que es un error psicopatologizar gratuitamente el primer elemento, ya que sus determinantes van mucho más allá del individuo aislado. Sin embargo, las guerras y los desastres naturales producen secuelas que sí son de interés y competencia psicológica, y la epidemiología ha realizado importantes esfuerzos en este campo..$^{21,22}$ Del otro lado de la moneda, tenemos la presencia de individuos claramente psicóticos responsables de muchos actos de violencia, en donde la psicopatología puede contribuir a diseñar programas de intervención más adecuados. ${ }^{23}$

\section{Contribuciones de la epidemiología al estudio de los trastornos mentales}

La epidemiología ha hecho contribuciones importantes para el conocimiento de la distribución, frecuencia y factores determinantes de los trastornos mentales en general, pero en particular ha sido muy relevante para el desarrollo de la psicopatología clínica.

Aunque una parte importante de la actividad epidemiológica contemporánea se enfoca a la búsqueda de marcadores biológicos y genéticos de exposición, ${ }^{24,25}$ no hay duda que el desglose que los individuos hacen acerca de lo que habitualmente comen, fuman, se ejercitan, etcétera, seguirá siendo material irremplazable para el trabajo epidemiológico. Desde hace años los estudios epidemiológicos hacen uso de cuestionarios (por autorreporte o por entrevista cara a cara) para obtener la información sobre factores de exposición. Han sido los sociólogos y los psicólogos, apoyados por la experiencia de la psicometría, los que más han aportado metodológicamente a esta tarea, discutiendo aspectos tales como la construcción de escalas, cuestionarios, cédulas de entrevista, etcétera; la validez y confiabilidad de las mediciones de los daños y la exposición; e inclusive la misma definición de los padecimientos investigados. ${ }^{26}$ Este conocimiento ha sido una importante aportación, tanto para la epidemiología en general como para el área de salud mental y la investigación clínica psiquiátrica. Esta última ha venido sufriendo una transformación notable en las últimas décadas, con una aceptación cada vez mayor del uso de instrumentos estandarizados para la definición de los casos de trastornos mentales, tanto en escenarios clínicos como comunitarios. ${ }^{27}$

Sin embargo, es en el campo específico de la psicopatología en donde la epidemiología de los trastornos mentales ha hecho las contribuciones más notorias, señalando al clínico las limitaciones de los casos que atienden en los servicios especializados, poniéndolos en relación con la población de donde provienen y completando el cuadro clínico de algunos padecimientos mentales, especialmente aquellos para los que la población solicita muy infrecuentemente los servicios especializados. Además, por supuesto, la mayoría de lo que sabemos sobre prevención en el área de los trastornos mentales proviene de la aportación epidemiológica.

Desde los primeros libros de texto de epidemiología psiquiátrica ${ }^{28}$ se ha reconocido que existe un gran "fenómeno de iceberg en los trastornos mentales": son muy pocos los casos que llegan a la atención especializada y hay una gran cantidad de casos que permanecen en la comunidad sin tratamiento, incluso con 
trastornos de tipo psicótico. El ejemplo más reciente de este fenómeno lo podemos observar en los trabajos del Epidemiologic Catchment Area (ECA), una investigación de seguimiento prospectivo realizada en varias ciudades de Estados Unidos de América (EUA), en donde se entrevistaron alrededor de 13400 sujetos. Según este trabajo, ${ }^{29}$ se encontró que sólo $9 \%$ de los casos de depresión, $14 \%$ de los casos de esquizofrenia y $6 \%$ de los casos de abuso de sustancias habían consultado con un especialista de los servicios de salud.

Pero la epidemiología de los trastornos mentales ha ido un paso más allá de esta constatación, si consideramos el ejemplo de lo sucedido con el síndrome de dependencia al alcohol. La investigación actual de este padecimiento considera el trabajo de Jellinek ${ }^{30}$ como parteaguas al formular su caracterización del cuadro clínico del alcoholismo, para lo cual se valió de los expedientes de más de 2000 miembros masculinos de la agrupación Alcohólicos Anónimos. El análisis de esta muestra de alcohólicos terminales en tratamiento y recuperación sugería que las personas en etapas iniciales e intermedias del alcoholismo progresarían irremediablemente hacia las etapas terminales del padecimiento, y que una vez allí sólo muy pocos lograban recuperarse. A partir de los trabajos realizados con muestras epidemiológicas, realizadas en la comunidad (y no sólo en pacientes clínicos) se pudo observar que los alcohólicos en tratamiento son sólo una pequeña porción de los casos y que su perfil tiende a ser diferente de los alcohólicos en la comunidad. Estos últimos tienden a ser más jóvenes, sus problemas son más aislados y se asocian entre sí menos que los problemas de los alcohólicos clínicos. Además, los estudios longitudinales de los alcohólicos en comunidad mostraban que los problemas que reportaban tendían a disminuir con el tiempo, y varios sujetos eran capaces de presentar transiciones entre episodios de enfermedad y franca recuperación voluntaria, negando la idea de que el tránsito a fases terminales de alcoholismo era un patrón general y que sólo muy pocos sujetos eran capaces de retroceder en la carrera alcohólica. Robin Room denominó a este fenómeno -a la diferencia entre el alcoholismo descrito en la clínica y el que se observa en la comunidad-como "los dos mundos del alcoholismo" ${ }^{31}$ Investigaciones realizadas también en la década de los setenta en veteranos de EUA de la guerra de Vietnam llegaron a conclusiones similares a este respecto: sujetos con problemas de dependencia a la heroína en Vietnam eran capaces de una franca recuperación en forma espontánea al regresar a sus hogares, cuestionando el concepto de irreversibilidad de las series clínicas para esta sustancia. ${ }^{32}$
En forma paralela, la casi totalidad de lo que sabemos sobre el proceso de búsqueda de atención especializada en los problemas psiquiátricos, así como los determinantes de la selección entre alternativas de tratamiento, provienen de estudios epidemiológicos realizados en la población general, que es aquella en la que se han investigado los patrones de búsqueda de atención especializada. ${ }^{6}$ Asimismo, si se considera que el proceso de prevención debe iniciarse no con la identificación de los casos clínicos (que ya presentan un cuadro clínico completo claramente definido) sino en aquellos que están en etapas previas del padecimiento, ${ }^{33}$ es claro que sólo mediante estudios epidemiológicos puede tenerse acceso a estos últimos sujetos.

El tema de la factibilidad de la prevención de los trastornos mentales está sujeto a una gran discusión. ${ }^{34,35}$ Weich ha sugerido que para avanzar en la prevención primaria de los trastornos mentales hace falta un mayor énfasis en el nivel poblacional, así como estudios longitudinales que avancen en la identificación de los factores de riesgo, y sugieran formas económicas de intervención, un refinamiento de las metodologías de evaluación de las intervenciones y un mejor conocimiento de las formas de movilizar a las comunidades. ${ }^{36}$ Aquí, como hemos señalado al inicio, es más práctico no hablar de salud mental en general sino especificar el problema o trastorno mental del que se habla, y analizar las particularidades del caso. Un ejemplo reciente de una intervención epidemiológica de este tipo puede observarse en la epidemiología de la farmacodependencia, con los trabajos de Holder y colaboradores sobre la prevención de accidentes y muertes relacionadas con el alcohol en tres comunidades de California, llevado a cabo por un periodo de cinco años. Este proyecto incluyó una serie de intervenciones tales como la movilización comunitaria, un componente de servicio responsable de venta, uno dirigido a la detección y reducción del consumo en conductores, y otras restricciones en el acceso al alcohol. Los resultados sugieren una disminución de $10 \%$ en los accidentes de tránsito en las comunidades que tuvieron la intervención, así como reducciones importantes en los otros componentes. ${ }^{37,38}$ Este es un excelente ejemplo de intervención exitosa, basada en una gran variedad de información epidemiológica referida a diferentes variables y proponiendo distintos niveles de intervención. Aunque estas intervenciones son costosas, puede demostrarse que su implantación es muy económica si se considera el ahorro que, a la larga, significan para los sistemas sanitario, legal y productivo. ${ }^{1}$ Intentos más limitados de aplicación de modelos preventivos existen también en la epidemiología del suicidio. En este 
caso, el problema es más complejo dada la diversidad de elementos al interior de la epidemiología del suicidio (ideas, planes, intentos, suicidio consumado) y el hecho de que la prevención de uno de los principales factores asociados al mismo (los trastornos mentales) es en sí mismo materia de debate. ${ }^{39}$

En un emotivo texto, Anthony y Van Etten ${ }^{20}$ defienden enfáticamente la necesidad de que la epidemiología psiquiátrica enfrente abiertamente los retos de la prevención. Siguiendo algunos ejemplos históricos -como la prevención de las manifestaciones psiquiátricas de la pelagra, la prevención de los efectos secundarios de la internación prolongada de los enfermos mentales, o la prevención de trastornos antisociales en niños con trastornos de conducta- ${ }^{40}$ estos autores plantean que en esta área, al igual que en otras de la epidemiología, lo que se necesita son epidemiólogos con orientación psicológica y psiquiátrica dedicados a encontrar causas específicas o mecanismos causales específicos para trastornos mentales específicos. Una vez en posesión de estos hallazgos se deben traducir a preguntas concretas de investigación preventiva y a la elaboración de ensayos comunitarios. Un ejemplo exitoso de esta combinación son los trabajos de Holder descritos anteriormente. ${ }^{37,38}$

\section{La nosología de los trastornos mentales y su medición epidemiológica}

Evolución de los estudios y de la medición de los trastornos mentales

Se ha clasificado a la epidemiología de los trastornos mentales en tres generaciones. ${ }^{11}$ La primera fase consistió en estudios centrados en el uso de registros e informantes clave para determinar la prevalencia comunitaria de trastornos mentales, más allá de los meros registros hospitalarios; la segunda fase, posterior a la Segunda Guerra Mundial, utilizó por primera vez entrevistas individuales a muestras comunitarias. La inmensa mayoría de los estudios de la segunda generación se basaron en instrumentos de medición que sólo permiten ubicar al sujeto en un continuo de salud-enfermedad mental, con poca especificidad, midiendo elementos generales de tensión psíquica que Dohrenwend ha llamado "desmoralización". La tercera generación de estudios inicia a fines de los años setenta, cuando los instrumentos de medición epidemiológica utilizados ya permiten detectar trastornos mentales específicos. A continuación presentamos los puntos centrales de la segunda y tercera generación de la epidemiología psiquiátrica, haciendo hincapié en los cambios de medición de los trastornos mentales.
Un resumen de las características principales de los instrumentos que se mencionan en el texto se puede consultar en Murphy ${ }^{6}$ y en Sartorius y Janca. ${ }^{41}$

Los primeros estudios que intentaron medir la frecuencia con que ocurrían los trastornos mentales en la población general fueron investigaciones exhaustivas de áreas geográficas delimitadas, cuya comparabilidad con resultados provenientes de otros estudios era limitada debido a las variaciones en los instrumentos y métodos empleados. Destacan los estudios llevados a cabo en la década de los cincuenta en los condados de Stirling, en Canadá, ${ }^{42}$ y en Midtown, Manhattan, ${ }^{43}$ que pretendieron probar hipótesis etiológicas específicas acerca de la relación causal de los factores socioambientales en la ocurrencia de los trastornos mentales.

En esta época se inició el estudio de la población que acudía a la práctica médica general. ${ }^{44,45}$ Se utilizaron instrumentos de tamizaje que pretendían identificar posibles casos con afección mental para ser confirmados en etapas posteriores, utilizando entrevistas clínicas. Estos instrumentos se construyeron bajo el supuesto de que era posible identificar elementos comunes en la mayor parte de los trastornos mentales menores, aquellos vistos con más frecuencia en la práctica médica general (sin incluir al alcoholismo y la dependencia a otras drogas). Las estimaciones que se derivaron de su utilización incluyeron una cantidad importante de "falsos positivos", esto es, de personas que al ser entrevistadas en una segunda vez no fueron confirmadas como casos. La idea de contar con instrumentos que valoraran la presencia de elementos comunes a la enfermedad mental fue cediendo paso a una nueva concepción que hizo hincapié en la diferenciación de las enfermedades mentales como entidades independientes, dando además lugar a la posibilidad de ocurrencia de co-morbilidad. ${ }^{46}$ Un cambio fundamental fue el observado en la propia evolución de la nosología psiquiátrica, con la aparición del Diagnostic and Statistical Manual of Mental Disorders (DSM), en EUA, y de la CIE-10, lo que permitió un mejor desglose de los síntomas individuales que componen los cuadros diagnósticos. A partir de este momento se desarrollaron instrumentos auto-aplicables o aplicados por entrevistadores no clínicos entrenados, útiles para estudios de población general. ${ }^{47}$

A mitad de la década de los setenta se desarrolló un nuevo instrumento, la cédula de Desórdenes Afectivos y Esquizofrenia (SADS, por sus siglas en inglés), que probó ser útil para evaluar pacientes psiquiátricos y controles sanos, y que fue utilizada en población general con resultados satisfactorios. ${ }^{48}$

Un siguiente paso se dio cuando se desarrolló el Present State Examination (PSE) ${ }_{1}^{49}$ para ser utilizado en 
un estudio transcultural entre EUA e Inglaterra. ${ }^{50}$ Este estudio demostró que las variaciones en el entrenamiento clínico de los psiquiatras de estas dos culturas jugaban un papel fundamental en la atribución de una prevalencia más elevada de esquizofrenia en EUA y de depresión en el Reino Unido. Este instrumento fue después utilizado en el estudio internacional de esquizofrenia, el primer estudio que utiliza un instrumento idéntico, traducido a diferentes lenguas. ${ }^{51}$

El PSE fue diseñado para ser aplicado por clínicos, haciendo muy cara su utilización en estudios de población general. Se encontró que cuando entrevistadores legos lo utilizaban en encuestas en población general tendían a calificar síntomas leves como positivos. Con el fin de evitar estos falsos positivos se construyó un programa especial llamado "índice de definición", que detecta y elimina a los falsos positivos antes de analizar los datos, utilizando el programa denominado catego.

En 1978, Robins ${ }^{52}$ inició el diseño de un instrumento, la Cédula de Entrevista Diagnóstica (DIS, por sus siglas en inglés), que pretendía superar las limitaciones del PSE. Dicho instrumento, que puede ser utilizado por encuestadores legos, investiga la presencia de síntomas en el curso de la vida y proporciona prevalencias de por vida, anual, de seis meses, un mes y dos semanas. Cuenta con un programa de cómputo fácil de usar, que contiene los algoritmos que permiten arribar a un diagnóstico a partir de la información vertida por el informante y sin intermediar el juicio del entrevistador. Así, se pueden obtener diagnósticos con base en los sistemas más importantes, tales como el DSMIII. ${ }^{53}$ Este instrumento se utilizó con éxito en lo que se constituyó un parteaguas de la investigación epidemiológica moderna, el ya mencionado Epidemiologic Catchment Area, que posteriormente sirvió de base a varios estudios, ${ }^{54}$ inaugurando la tercera generación de estudios epidemiológicos.

Este instrumento, el DIS, a pesar de ser largo -con una duración promedio de 40 minutos en entrevistas a personas más o menos asintomáticas y de 60 a $120 \mathrm{mi}-$ nutos cuando se le aplica a personas con afecciones mentales- probó tener bastante aceptación en la población general. Sin embargo, al aplicar estos instrumentos en diferentes escenarios culturales se han encontrado problemas con la utilización de criterios que son típicos de la población norteamericana, para la que fueron elaborados. ${ }^{55}$

Un siguiente paso en esta evolución de los instrumentos de medición epidemiológica de los trastornos mentales fue la elaboración del Composite International Diagnostic Interview (CIDI), una entrevista diagnóstica elaborada explícitamente para ser aplicada en estudios internacionales, ${ }^{56,57}$ desarrollado por un grupo de trabajo convocado por la OMS y los Institutos Nacionales de Salud de Estados Unidos de América. Este grupo de trabajo utilizó el DIS como instrumento base, haciéndole adaptaciones para ser usado en el contexto internacional, incluyendo a países en desarrollo. Además, se añadieron preguntas para obtener diagnósticos mediante la CIE-10. ${ }^{58}$ Este instrumento fue sometido a pruebas en varios países con diferente nivel de desarrollo y antecedentes culturales, con resultados satisfactorios. ${ }^{59}$

Posteriormente, Kessler ${ }^{60}$ modificó el CIDI y lo utilizó en la Encuesta Nacional de Co-morbilidad, realizada en EUA, primera investigación en ese país que aportó estimaciones de los principales trastornos mentales, representativa en el ámbito nacional. Dicho investigador estaba interesado en utilizar los resultados de la encuesta para reconstruir la historia de los trastornos, por lo que dio mayor importancia a la obtención confiable de información sobre la edad de la primera aparición de síntomas de trastornos mentales. También se propuso disminuir el índice de no respuesta por lo que cambió la forma de presentar las preguntas. Así, en primer lugar, se obtiene información sobre la presencia o ausencia de cada uno de los síntomas que conforman un diagnóstico y, en un segundo momento, se hace el interrogatorio que permitiera eliminar aquellos síntomas que no son producto de alguna enfermedad o condición no atribuible a un trastorno mental.

Más recientemente, la OMS revisó también su instrumento original (el CIDI) dando lugar a diversas colaboraciones transculturales. ${ }^{61,62}$ Una versión modificada y adaptada para su aplicación en los cinco continentes formó parte de la iniciativa de la OMS para el año 2002,' que realizó estudios simultáneos para detectar la prevalencia de trastornos mentales en los cinco continentes utilizando metodologías e instrumentos similares.

A la par de la medición con fines epidemiológicos de estudios en la población general se desarrolló también una cédula de evaluación clínica conocida como SCAN (Schedules for Clinical Assessment in Neuropsychiatry) ${ }^{63}$ que es la contraparte clínica del DIS. El SCAN se basó en el PSE al que se le incluyeron preguntas para incluir un mayor número de trastornos y para que permitiera arribar a un diagnóstico de acuerdo con el DSM-III-R y la CIE-10. Ambos instrumentos, el CIDI y el SCAN, son calificados por medio de computadora, y pueden ser aplicados utilizando este recurso.

Un tercer instrumento es el IPDE o Examen Internacional de Desórdenes de la Personalidad. El objetivo de este instrumento es evaluar el eje II del DSM-III-R y 
los desórdenes de la personalidad como son definidos por la CIE-10. ${ }^{64}$ Esto ha resultado ser una tarea particularmente difícil, puesto que la propia definición de los trastornos de personalidad pone en duda los resultados que se puedan lograr en una entrevista con estos sujetos.

Por otra parte, en los últimos años han proliferado los instrumentos para la medición de discapacidad. ${ }^{41}$ La Clasificación Internacional del Funcionamiento y la Discapacidad, en su segunda versión (International Classification of Functioning and Disability o ICIDH-2), ${ }^{65}$ es el resultado de la revisión de la Clasificación Internacional del Deficiencias, Discapacidad y Minusvalía (International Classification of Impairments, Disabilities, and Handicaps, o ICIDH) que funcionó durante más de 20 años. ${ }^{66}$ Esta clasificación del funcionamiento y la discapacidad fue concebida como una herramienta de comunicación entre diferentes disciplinas y es útil para valorar situaciones clínicas, descripciones funcionales, datos cuantificables y códigos numéricos independientes del idioma de origen. Se basa en el concepto de que la enfermedad y la discapacidad son constructos diferentes, que pueden ser medidos independientemente. El nivel de discapacidad no debe ser visto automáticamente como un indicador de la severidad de la enfermedad. Así, se puede tener una enfermedad con diferentes grados de severidad (diabetes, depresión, etcétera) y las discapacidades no estar relacionadas necesariamente con la severidad de las enfermedades subyacentes, sino con factores del medio ambiente que limitan tanto la actividad como la participación de los enfermos, y que pueden variar de una sociedad a otra. Se ha desarrollado también un Instrumento de Evaluación de Discapacidades (WHO-DAS) por medio del cual se evalúan las consecuencias de la enfermedad de manera estructurada y de acuerdo con la segunda versión de la ICIDH (comúnmente denominada como ICIDH-2) ${ }^{65}$

\section{Diseños de estudio y métodos de análisis más usuales de los trastornos mentales}

La investigación epidemiológica moderna de los trastornos mentales utiliza el arsenal metodológico común a otras áreas de la epidemiología, tales como los diseños transversales, los estudios de casos y controles, y los de cohorte. ${ }^{67}$ Una revisión de los principales diseños epidemiológicos y de su aplicación en la epidemiología de los trastornos mentales puede ser consultada en Zahner y colaboradores. ${ }^{68}$ Un ejemplo actual de estudios de corte transversal se puede consultar en Merikangas y colaboradores ${ }^{61}$ quienes reportan resultados de encuestas representativas de la pobla- ción de EUA, México, Alemania, Canadá y los Países Bajos, utilizando el ya mencionado CIDI como instrumento de recolección de datos. Dichos autores presentan información sobre la prevalencia de trastornos de ansiedad, afectivos, uso de sustancias tóxicas y trastornos de la personalidad, así como datos sobre el proceso de co-morbilidad entre los trastornos secundarios al uso de sustancias y los demás. Un ejemplo actual de estudio prospectivo se puede encontrar en varios de los reportes del ECA, como los de Eaton y colaboradores $^{69}$ sobre el surgimiento y recuperación de trastornos de pánico en una muestra representativa de la población general de Baltimore, formada por 3841 individuos seguidos por más de 10 años. Un ejemplo reciente de un estudio de casos y controles puede consultarse en la literatura sobre el suicidio, utilizando la metodología de autopsia psicológica practicada por Brent y colaboradores ${ }^{70}$ y por Shah y colaboradores. ${ }^{71}$ Estos últimos compararon la prevalencia de armas de fuego en las residencias de 36 víctimas de suicidio con armas de fuego y en controles comunitarios, pareados por edad, sexo y escuela de la víctima, aportando datos muy relevantes para fundamentar la legislación sobre la compra y posesión de armas de fuego en EUA. ${ }^{72}$ Respecto a los estudios ecológicos, la epidemiología de los trastornos mentales ha producido una gran cantidad de trabajos utilizando este tipo de estudios, especialmente en lo referente al impacto del consumo per cápita de bebidas alcohólicas y problemas tales como la cirrosis hepática ${ }^{73}$ y los actos criminales. ${ }^{74} \mathrm{Si}$ nos enfocáramos a un problema en particular, como el suicidio, también podríamos encontrar ejemplos de investigaciones que muestran el despliegue de todos los diseños epidemiológicos generales. ${ }^{5}$

La revisión de otros campos de especialidad muestra resultados similares en cuanto a la amplitud de los diseños de investigación y las técnicas epidemiológicas empleadas. Ejemplos de ellos pueden consultarse en los estudios sobre la esquizofrenia, ${ }^{75}$ la influencia de los eventos de vida sobre la depresión ${ }^{76}$ o la epidemiología de los trastornos mentales en población infantil. Como comenta Rutter, ${ }^{77}$ la epidemiología en esta área ya ha dejado de ser un mero ejercicio de contabilidad de cabezas, y ha pasado a ser un instrumento que permite responder a una multiplicidad de preguntas en el área del tratamiento, las políticas de salud y las prácticas profesionales, en general.

Pero la metodología para estudiar los trastornos mentales se ha nutrido también de otras fuentes. A los diseños epidemiológicos generales, fruto de la tradición de la salud pública y su entrecruzamiento con la psicopatología, se podrían agregar todas aquellas aproximaciones provenientes de la tradición de la in- 
vestigación en psicología clínica, psicología social y de psicometría -a la que nos referimos con anterioridadasí como las aportaciones de las ciencias sociales, que en este campo han tenido una importancia histórica innegable. ${ }^{18}$ No es este el lugar para hacer una revisión de las aproximaciones metodológicas de otras disciplinas, pero debemos mencionar que el uso de modelos estructurales y variables latentes es mucho más generalizado en el área de la epidemiología de los trastornos mentales que en casi cualquier otra área de la epidemiología. ${ }^{7879} \mathrm{La}$ aplicación de estas técnicas se va haciendo mucho más generalizada en estudios de epidemiología genética en el campo de los trastornos mentales ${ }^{80,81}$ y es esperable que su aplicación pronto se generalice a otras áreas de la epidemiología. El resultado de esta amalgama de tradiciones analíticas es un campo complejo y dinámico, del que se pueden esperar muchas aportaciones en el futuro próximo.

No obstante los avances en la aplicación de los diseños epidemiológicos en el estudio de los trastornos mentales, hay coincidencia también de que en pleno siglo XXI la mayoría de los trabajos en este campo aún son de tipo descriptivo, principalmente trasversales, y referidos a la prevalencia de los trastornos mentales. ${ }^{8}$ Incluso varios de los estudios de epidemiología genética en psiquiatría son, desde el punto vista estrictamente epidemiológico, estudios transversales que obtienen mediciones de marcadores genéticos combinados con información retrospectiva de otros factores de exposición y comparten, por lo tanto, todas las limitaciones y sesgos de los estudios transversales tradicionales. ${ }^{82,83}$

Varias razones explican este hincapié en los estudios transversales. En primer lugar, una gran parte de los trastornos mentales se componen de síntomas sobre los cuales es sumamente difícil especificar la fecha de primera aparición; también es frecuente que los pacientes se presenten a los servicios especializados hasta muy avanzado el proceso mórbido, lo que hace que la integración de síndromes específicos sea difícil y el uso de medidas de prevalencia se convierta en la forma más sencilla de estudio. En segundo lugar, muchos de los padecimientos psiquiátricos son raros y recurrentes, de manera que el uso de medidas de prevalencia (especialmente la prevalencia lápsica, y la prevalencia de alguna vez en la vida) es útil porque permite captar un número mayor de casos, incluso cuando están en remisión temporal al momento de la entrevista.

Una discusión sobre las dificultades teóricas y prácticas del uso de medidas de incidencia en la investigación epidemiológica moderna de los trastornos mentales se puede consultar en Eaton ${ }^{84}$ quien muestra la historia natural de la psicopatología para un trastorno específico. No obstante, las grandes dificultades derivadas de la medición y el seguimiento de los trastornos mentales han hecho que se carezca hasta el momento de estándares aceptados de la incidencia de la mayoría de los trastornos mentales en población general, ni siquiera de los más comunes. De acuerdo con el mismo Eaton, si se establece como criterio de un estudio prospectivo adecuado aquel que contenga por lo menos 5000 años persona de observación -para obtener estimaciones estables de los trastornos mentales más comunes- en 1995 sólo se habían realizado cuatro estudios de este tipo. ${ }^{84}$

En forma alternativa a los estudios de seguimiento, algunas investigaciones han tratado de establecer las edades de inicio de los trastornos mentales en forma retrospectiva. No obstante su atractivo para avanzar en la búsqueda de causas, la información retrospectiva sufre de posibles sesgos, como sucede cuando existen problemas de memoria en las personas estudiadas, cuando se estudia sólo a los sobrevivientes del suceso de interés o, quizá más importante aun, cuando se investiga la edad de inicio del trastorno, pero no el momento de aparición de los factores de riesgo en forma igualmente retrospectiva (como, por ejemplo, si el padre estaba desempleado al momento cuando se reportan los primeros síntomas de depresión) ${ }^{85}$

Sin duda, los trastornos psiquiátricos plantean problemas muy serios de medición para la aplicación de diseños longitudinales, pero el entrenamiento epidemiológico moderno nos puede ayudar a solucionar algunos problemas añejos en el área. Por ejemplo, la discusión sobre si la relación entre trastornos mentales y estatus socioeconómico se debe a un proceso de causalidad social (adversidad y tensión) o a un proceso de selección social (movilidad hacia abajo de personas con predisposición genética) adquirió nuevas luces con el trabajo reciente de Dohrenwend y colaboradores.$^{86}$ Estos autores aprovecharon la migración que comenzó hacia Israel alrededor de 1948, migración formada por judíos europeos, étnicamente blancos, y judíos africanos y del medio oriente, étnicamente negros y sujetos a prejuicios y discriminación. Esta diferencia étnica es un antecedente claro, y no puede cambiar en el tiempo, como lo puede el caso del nivel socioeconómico. Se presenta así, un ejemplo de estudio cuasiexperimental. De la misma manera, este grupo de investigadores ha tratado de analizar los diferenciales de mortalidad en la población hispana de EUA, analizando separadamente las tasas de mortalidad de la población cubana (que no puede regresar a morir a Cuba), la población de Puerto Rico (que aunque regrese a 
morir a Puerto Rico tiene su muerte contabilizada en EUA) y la población anglosajona, manteniendo controlados los efectos de la posible migración sana. ${ }^{87}$ Finalmente, este mismo grupo ha realizado investigaciones longitudinales sobre las teorías de la causalidad o selección social de los trastornos mentales en Estados Unidos, llegando a resultados muy similares a los encontrados en la investigación realizada en Israel. ${ }^{88}$ Como se ve en este ejemplo, un grupo de investigadores ha subsanado algunos de los problemas de la investigación epidemiológica en el campo de la salud mental haciendo uso de modernas técnicas de medición psicométrica. Estos ingeniosos diseños permiten mantener constantes algunas características complejas que naturalmente cambian con el tiempo y que no podrían ser medidas usando los tradicionales diseños longitudinales.

\section{Perspectivas futuras para la epidemiología de los trastornos mentales}

La epidemiología de los trastornos mentales se enfrenta a enormes retos en el nuevo milenio. Debe hacer frente a un panorama epidemiológico complejo y cambiante. En éste, los padecimientos psiquiátricos aparecen en un lugar sumamente importante y hay más y más personas interesadas por el desarrollo de esta disciplina. ${ }^{10}$ En los últimos años, muchos investigadores han expuesto sus opiniones y recomendaciones sobre la dirección que deben de tomar los nuevos estudios de la epidemiología psiquiátrica, con opiniones no siempre coincidentes. ${ }^{89-92}$ Una discusión pormenorizada de estos planteamientos va más allá de los límites de esta somera revisión a nuestro campo. Sin embargo, podemos distinguir los siguientes rubros que parecen centrales en el desarrollo epidemiológico venidero.

\section{Medición de los trastornos mentales y de los factores de riesgo}

La epidemiología psiquiátrica está lejos de haber solucionado los problemas que recurrentemente se representan durante la definición de los trastornos mentales, ni podemos pensar que, por sí sola, lo pueda hacer. No han sido despreciables los avances experimentados en el campo de la clínica de los trastornos mentales en los últimos 20 años, así como en la forma como los psiquiatras y psicólogos clínicos se pueden entender y poner de acuerdo sobra las características básicas de los principales cuadros clínicos. La epidemiología ha hecho uso de estos avances y ha logrado impactar en la misma clínica. No obstante, diferentes aspectos de la medición de los trastornos mentales en la comunidad son urgentes. Algunos de ellos tienen que ver con aspectos básicos de estos trastornos, y deben desarrollarse de tal manera que las mediciones en comunidad permitan influir en la definición de las necesidades de atención clínica. ${ }^{30,90}$ Este es el caso, por ejemplo, de la medición de la discapacidad asociada a los trastornos mentales.

Asimismo, se espera disolver las diferencias entre la tradición clínica y la tradición psicométrica de los trastornos mentales. Otros aspectos tienen que ver con avances instrumentales, como la introducción de entrevistas por computadoras y la interfase con la entrevista clínica como validación de las mediciones comunitarias. Podemos esperar importantes avances en estas áreas en la próxima década. Por otro lado, muchos de los factores de riesgo para los trastornos mentales son de los llamados distales, es decir, de un pasado remoto. Cómo obtener información de estos factores en personas que sufren enfermedades que debilitan la memoria y el juicio objetivo es un problema fundamental que amerita atención especial.

\section{Diseño y métodos de muestreo más eficientes}

Ya hemos mencionado antes que la epidemiología psiquiátrica sigue siendo restringida en cuanto a los diseños de estudio que despliega con mayor frecuencia. Aunque hay elementos para pensar que el propio objeto de estudio plantea limitaciones al respecto, también hemos señalado que es posible, con entrenamiento en la metodología epidemiológica, solventar algunos de estos problemas y plantear acercamientos novedosos e ingeniosos a los problemas de nuestra área. En la actualidad es indispensable superar la metodología basada en la aplicación de encuestas epidemiológicas de prevalencia y orientar las prioridades hacia la investigación causal. ${ }^{93,94}$ De hecho, es poco probable que, después de la iniciativa de la OMS para el año 2000, se dediquen fondos significativos a nuevos estudios de prevalencia. Es posible, y deseable, que en este nuevo siglo resurjan aproximaciones de mayor alcance analítico, con énfasis en los diseños de casos y controles y en aproximaciones más económicas que los diseños longitudinales tradicionales. ${ }^{93}$ En la medida en la que ha quedado claro que muchos de los trastornos mentales tienen rutas de origen tempranas, los diseños de intervención en poblaciones de adolescentes deberán de incrementarse también en el futuro próximo. Estudios transculturales y de inmigrantes plantean un reto importante en el nuevo milenio, el cual parece que estará caracterizado por una gran movilidad humana. 
3. Relación entre la investigación biológica, genética, las disciplinas sociales y la epidemiología

La epidemiología se encuentra en un momento de reflexión en relación con los avances de la genética y la contribución de ésta al entendimiento del proceso causal en diferentes enfermedades. La fundamentación del campo de la epidemiología genética es una realidad, y cada vez son más los estudios en este campo. Hasta qué punto las promesas de la genética en el campo específico de los trastornos mentales se van a cumplir en un futuro próximo, es materia de debate. Algunas de las mentes más brillantes de nuestra área han planteado que el futuro de la epidemiología de los trastornos mentales provendrá de la epidemiología genética. ${ }^{93}$ Aunque hasta ahora estas promesas parecen ser exageradas o, cuando menos, prematuras, no hay duda de que se esperan muchos avances y nuevas formas de unión entre la epidemiología genética y la investigación psicopatológica. Afortunadamente, pocos creen que esta combinación deba re-abrir la discusión entre nature-nurture, y confían en que la discusión se dirija desde el inicio hacia la comprensión de la extraordinariamente compleja relación que existe entre los genes y el ambiente, más que al encuentro de la preminencia de unos sobre el otro, o viceversa. ${ }^{94}$ En esta búsqueda, los diseños y la teoría epidemiológica tendrán un papel determinante, como lo han tenido en la comprensión de las relaciones entre la salud mental individual y las condiciones sociales colectivas. ${ }^{95}$ Por este motivo, en la medida en la que avance nuestro conocimiento de la genética de los principales trastornos mentales, esperamos un avance concomitante en la comprensión que juegan los factores sociales en la producción de estos mismos fenómenos. ${ }^{25,96}$

\section{Interfase entre la epidemiología y la evaluación de los tra- tamientos y los servicios}

De lo revisado hasta aquí, queda claro que una limitación importante de la epidemiología de los trastornos mentales ha sido la escaza interfase con los servicios de salud. ${ }^{97}$ En primer lugar, no está del todo claro que la estrategia utilizada hasta ahora para la realización de los estudios en comunidad tenga una traducción inmediata para los servicios clínicos. ${ }^{98}$ Por tal motivo, en segundo término, es necesario orientar las investigaciones futuras hacia la promoción de este acercamiento. ${ }^{27,99}$ Por otro lado, un área que hasta ahora ha estado claramente limitada a la psicopatología, en general, es la evaluación de las estrategias terapéuticas, en especial las diferentes formas de psicoterapia. En la medida en la que los trastornos mentales adquieren mayor peso en el perfil epidemiológico, se exigirá que se fundamente con mayor rigor la eficacia y eficiencia de los diferentes tipos de tratamiento y cómo estos se pueden traducir en ganancias económicas para las instituciones y los pacientes individuales. ${ }^{100} \mathrm{La}$ evaluación de los servicios de salud mental se convertirá, sin duda, en un campo muy activo durante el siglo XXI.

\section{Referencias}

1. Murray C, López A.The global burden of disease. Boston (MA): Harvard School of Public Health. World Health $O$ rganization. World Bank. Harvard University Press; 1996.

2. Kessler RC. The W orld Health O rganization International

Consortium in Psychiatric Epidemiology (ICPE): Initial work and future directions - The N APE Lecture 1998. Nordic Association for Psychiatric Epidemiology. Act Psychiatr Scand 1999;99(1):2-9.

3. Ustun TB.The global burden of mental disorders. Am J Public Health 1999;89(9): 1315-1318.

4.Anthony JC, Eaton W W, Henderson AS. Psychiatric epidemiology. Epidemiol Rev 1995; 17(1):1-8.

5. Borges $G$, Anthony JC, Garrison CZ. Methodological issues relevant to epidemiologic investigations of suicidal behaviors of adolescents. Epidemiol Rev 1995;17(1):228-239.

6. Murphy JM. Diagnostic schedules and rating scales in adult psychiatry. En:Tsuang MT,Tohen M, Zahner GEP, Ed.Textbook in psychiatric epidemiology. N ueva York (N Y):John W iley Liss; 1995.

7. Babor TF, Brown J, D el Boca FK.Validity of self-reports in applicd research on addictive behaviors: Fact or fiction? Behav Assess 1990; 12(1):5-31.

8. Kessler RC, W ittchen HU,A belson J, Zhao S. Methodological issues in assessing psychiatric disorders with self-reports. En: Stone AA, Turkkan JS, Bachrach CA, Jobe JB, Kurtzman HS, C ain VS, ed. The science of selfreport: Implications for research and practice. Mahwah (N J): Lawrence Erlbaum Associates Publishers; 2000: 229-255.

9.A merican Psychiatric Association. Diagnostic and statistical manual of mental disorders, 4th Ed. (DSM-IV). W ashington DC:A merican Psychiatric Association; 1994.

10. W orld Health 0 rganization. Mental and behavioral disorders, diagnostic criteria for research: International classification of diseases, injuries and causes of death, 10th. revision. G inebra, Suiza:W HO ; 1990. 11. D ohrenwend BP, D ohrenwend BS. Perspectives on the past and future of psychiatric epidemiology.The 1981 Rema Lapouse Lecture.Am J Public Health 1982;72(11): 1271-1279.

12. Lerman-G arber I, Barrón-U ribe C, C alzada-León R, Mercado-Atri M, Vidal-Tamayo R, Q uintana $S$ et al. Emotional dysfunction associated with diabetes in Mexican adolescents and young adults with type-1 diabetes. Salud Publica Mex 2003;45:13-18.

13. W ittchen HU. Epidemiological research in mental disorders: Lessons for the next decade of research-The N APE Lecture 1999. Acta Psychiatr Scand 2000;101(1):2-10.

14. Grant BF. Epidemiology.Annu Rev Addict Res Treatment 1993;3: 71-86.

15.Anthony JC. Epidemiology of drug dependence. En: Galanter M, Kleber HD, ed. Textbook of substance abuse treatment: 2nd Edition. W ashington, DC:A merican Psychiatric Press; 1999: 47-58.

16. Medina-Mora ME. Surveys that work and what they show in Mexico. J Addict D is 1991;11(1):23-32. 
17. Lazcano-Ponce EC, Hernández-Avila M. La epidemia de tabaquismo. Epidemiología, factores de riesgo y medidas de prevención. Salud Publica Mex 2002;44(S1):S1-S2.

18. Kessler RC. Sociology and psychiatry. En: Kaplan H, Sadock B, ed. Comprehensive textbook of psychiatry. Seventh Edition. Baltimore (MD):W illiams and W ilkins; 1998.

19.Vander-Stoep A, Beresford SA, W eiss N S, McKnight B, Cauce AM, Cohen P. Community-based study of the transition to adulthood for adolescents with psychiatric disorder.Am J Epidemiol 2000; 152(4): 352-362.

20.Anthony JC, Van-Etten ML. Epidemiology and its rubrics. En: Bellack A, Hersen M, Ed. Comprehensive clinical psychology.Vol. I. 0 xford (UK): Elsevier Science Publications; 1999:355-390.

21. Bromet E, D ew MA. Review of psychiatric epidemiologic research on disasters. Epidemiol Rev 1995;17(1):113-119.

22. N eugebauer R. Mind matters:The importance of mental disorders in public health's 21st century mission. Am J Public Health 1999;89(9): 1309-1311.

23. Buka S, Earls F. Early determinants of delinquency and violence. Health Aff (Millwood) 1993; 12(4):46-64.

24. Hunter DJ.The future of molecular epidemiology. Int J Epidemiol 1999;28(5):SI012-S1014.

25. Susser M. Should the epidemiologist be a social scientist or a molecular biologist? Int J Epidemiol 1999;28(5):S1019-S1022.

26. Susser M. Epidemiology in the United States after W orld W ar 11: The evolution of technique. Epidemiol Rev 1985;7:147-177.

27.W ittchen HU, Ustun TB, Kessler RC . Diagnosing mental disorders in the community. A difference that matters? Psychol Med 1999;29(5):1021-1027.

28. Cooper B, Morgan HG. Epidemiological psychiatry. Springfield (IL): Thomas; 1973.

29. Marino S, Gallo JJ, Ford D,Anthony JC. Filters on the pathway to mental health care. I. Incident mental disorders. Psychol Med 1995;25(6):1135-1148.

30. Jellinek EM. D isease concept of alcoholism. N ew Haven (CT): College and University Press; 1972:246.

31. Room R. Treatment-seeking populations and larger realities. En: Edwards $G, G$ rant M, ed. Alcoholism treatment in transition. London, England: Croom Helm; 1980:205-224.

32. Robins LN, Helzer JE, Davis DH. N arcotic use in Southeast Asia and afterward. An interview study of 898 Vietnam returnees. Arch $G$ en Psychiatry 1975;32(8):955-961.

33. Anthony JC. Putting epidemiology and public health in needs assessment: D rug dependence and beyond. En:Andrews $G$, Henderson S, ed. Unmet need in psychiatry: problems, resources, responses. N ueva York (N Y): C ambridge University Press; 2000:302-308.

34. Bums JM, Patton GC. Preventive interventions for youth suicide: $A$ risk factor-based approach. Aust N Z J Psychiatry 2000;34(3):388-407. 35. Martínez-Martínez KI, Carrascosa-Venegas C, Ayala-Velásquez H. Una estrategia de diseminación en la psicología: los talleres interactivos. Salud Publica Mex 2003;45(1):5-12.

36. W eich S. Prevention of the common mental disorders: a public health perspective. Psychol Med 1997;27(4):757-764.

37. Holder HO, Saltz RF, Grube JW, Voas RB, Gruenewald PJ,Treno AJ.A community prevention trial to reduce alcohol-involved accidental injury and death: 0 verview. Addiction 1997; 92 suppl 2: S155-S171.

38. Holder HO.Community policy and the prevention of alcoholinvolved trauma. En: 6th Meeting of Heads of Major Alcohol C entres: Proceedings; 1996 September 11-13,Tokyo, Japan: N ippon Foundation; 1997:61-72.

39. United $\mathrm{N}$ ations. Prevention of suicide. G uidelines for the formulation and implementation of national strategies. Nueva York (NY): United N ations; 1996.
40. Mrazck P, Haggerty RJ. Reducing risks for mental disorders: Frontiers for preventive intervention research. W ashington, $D C: N$ ational Academy Press; 1994.

41. Sartorius $N$, Janca A. Psychiatric assessment instruments developed by the W orld Health 0 rganization. Soc Psychiatry Psychiatr Epidemiol 1996:31 (2):55-69.

42. Leighton $\mathrm{O}, \mathrm{Mac}$ Millan $\mathrm{A}$, Hardin $\mathrm{H}$, Macklin $\mathrm{O}$, Leighton A. The character of danger. N ueva York (NY): Basic Books; 1963.

43. Srole L, Langner TS, Michael ST, O pler KM, Rennie T A. Mental in the Metropolis:The Midtown Manhattan Study. N uevaYork: McG raw-Hill; 1962.

44. Harding OT, D e A rango MV, Baltazar J, C liment CE, Ibrahim HH, Ladrido-Ignacio $\mathrm{L}$ et al. Mental disorders in primary health care:A study of their frequency and diagnosis in four developing countries. Psychol Med 1980; 10(2):231-241.

45. Goldberg 0 . The detection of psychiatric illness by questionnaire. Londres: 0 xford University Press;1972.

46. W eissman MM, Klerman GL. Epidemiology of mental disorders: Emerging trends in the United States. Arch Gen Psychiatry 1978;35(6):705-712.

47. García-G arcía E,Vázquez-Velásquez V, López-Alvarenga JC,ArcilaMartínez D.Validez interna y utilidad diagnóstica del Eating Disorders Inventory en mujeres mexicanas. Salud Publica Mex 2003;45:206-210 48. W eissman MM, Bruce ML, Leaf P, Florio LP, Holzer C III.Affective disorders. En: Robins $L N$, Regier $O A$, ed. Psychiatric Disorders in America. N ueva York (N Y): Free Press; 1991.

49.W ing J, Birley J, C o oper J, Graham P, Issacs A. Reliability of a procedure for measuring and classifying "present psychiatric state". Br J Psychiatry 1967; 113: 449-515.

50. C ooper J, Kendell E, G urland J, Sartorius N. Cross-national study of diagnosis of mental disorders in the general population. Acta Psychiatr Scand (Suppl) 1969;348(79):S157-S166.

51. W orld Health 0 rganization. The International Pilot Study of Schizophrenia. Vol. I. Ginebra:W HO 1973.

52. Robins L. Psychiatric epidemiology. Arch Gen Psychiatry 1978;35(6):697-702.

53. A merican Psychiatric Association. Diagnostic and statistical manual of mental disorders. 3rd Ed. (DSM-III).W ashington, DC:A merican Psychiatric Press; 1980.

54. Regier DA, Myers JK, Kramer M, Robins LN, Blazer DG, Hough RL et al. The N IMH Epidemiologic Catchments A rea Program. Historical context, major objectives, and study population characteristics. Arch Gen Psychiatry 1984;41(10):934-941.

55. Robins L. Cross-cultural issues in diagnosis. En: Hoppe S, Holtzman W H, Ed. Search for a common language in psychiatric assessment. Texas (TX):Texas W orld Health 0 rganization Collaborating C enter; 1994: 21-35.

56. W orld Health 0 rganization. Composite International Diagnostic Interview (CIDI), version 1.1.W ashington, DC:A merican Psychiatric Press; 1993.

57. Robins L,W ing J,W ittchen HU, Helzer JE, Babor TF, Burke J et al.The composite international diagnostic interview:An epidemiologic instrument suitable for use in conjunction with different diagnostic systems and different cultures. Arch Gen Psychiatry 1988;45( 12):10691077.

58. W orld Health 0 rganization. Mental health and behavioral disorders (including disorders of psychological development). En: International Classification of Diseases, 10th Rev. G inebra, Suiza:W HO ; 1992:311-387. 59. W ittchen H, Robins L, Cottler L, Sartorius N, Burker J, Regler D. Cross-cultural feasibility, reliability and sources of variance of the Composite International Diagnostic Interview (CIDI). Br J Psychiatry 1991: 645-653. 
60. Kessler RC, MCG ongale KA, Z hao S, N elson CB, Hughes M, Eshelman $S$ et al. Lifetime and 12-month prevalence of DSM-III-R psychiatric disorders in the United States. Arch Gen Psychiatry 1994:51:8-19.

61. Merikangas KR, Mehta RL, Molnar BE,W alters EE, Swedsen JD, A guilar-G axiola $S$ et al. Comorbidity of substance use disorders with mood and anxiety disorders: results of the International Consortium in Psychiatric Epidemiology.Addict Behav 1998;23(6): 893-907.

62. W orld Health 0 rganization. International C onsortium of Psychiatric Epidemiology. Cross-national comparison of mental disorders. Bull W orld Health 0 rgan 2000;78(4)413-426.

63. W orld Health 0 rganization. Schedules for $C$ linical Assessment in N europsychiatry (SCAN).W ashington DC:A merican Psychiatric Press; 1993.

64. W orld Health 0 rganization. International Personality Disorder Examination (IPDE).W ashington, DC:A merican Psychiatric Press; 1994.

65. W orld Health $O$ rganization. International Classification of Functioning and Disability. Beta 2. D raft for Field Trials (ICID H-2). Ginebra:W HO ; 1999.

66. W orld Health $\mathrm{O}$ rganization. International Classification of Impairments, Disabilities and Handicaps (ICIO H). Ginebra:W HO ; 1980. 67. Hernández-Avila M, G arrido-Latorre F, López-Moreno S. Diseño de estudios epidemiológicos. Salud Publica Mex 2000;42 (2): 144-154. 68. Zahner GE, H sieh CC, Fleming JA. Introduction to epidemiologic research methods. En:Tsuang MT,Tohen M,Zahner GEP, ed.Textbook in psychiatric epidemiology. N ueva York (N Y): John W iley-Liss; 1995. 69. Eaton W W, Anthony JC, Romanoski A, Tien A, G allo J, C ai G et al. 0 nset and recovery from panic disorder in the Baltimore Epidemiologic Catchment Area Follow-up. Br J Psychiatry 1998; 173:501-507.

70. Brent DA, Baugher M, Bridge J, Chen T, Chiappetta L.Age and sexrelated risk factors for adolescent suicide. J Am A cad C hild Adolesc Psychiatry 1999;38(12):1497-1505.

71. Shah S, Hoffman RE,W ake L, Marine W M. Adolescent suicide and household access to firearms in Colorado: Results of a case-control study. J A dolesc Health 2000;26(3): 157-163.

72. Lambert MT, Silva PS. An update on the impact of gun control legislation on suicide. Psychiatr Q 1998;69(2):127-134.

73. Skog 0 J. Risk function for liver cirrhosis from lifetime alcohol consumption. J Stud Alcohol 1984;45(3):199-208.

74. N orstrom T. Effects on criminal violence of different beverage types and private and public drinking.Addiction 1998;93(5):689-699.

75. Bromet EJ, Fennig S. Epidemiology and natural history of schizophrenia. Biol Psychiatry 1999; 46(7):871-881.

76. Kessler RC. The effects of stressful life events on depression.Ann Rev Psychol 1997;48:191-214.

77. Rutter M. Isle of W ight revisited:Twenty-five years of child psychiatric epidemiology. J Am Acad Child Adolesc Psychiatry 1989;28(5):633-653.

78. Gallo JJ,Anthony JC, Muthen BO.Age differences in the symptoms of depression:A latent trait analysis. J Gerontol 1994;49(6):251-264.

79. Muthen $\mathrm{BO}$, Muthen LK.The development of heavy drinking and alcohol-related problems from ages 18 to 37 in a US national sample. J Stud Alcohol 2000;61(2):290-300.

80.Van den Bree MB, Johnson EO, N eale MC, Pickens RW. Genetic and environmental influences on drug use and abuse/dependence in male and female twins. Drug Alcohol Depend 1998;52(3):231-241.
81. Kendler KS, Karkowski LM,W alsh D.The structure of psychosis: Latent class analysis of probands from the Roscommon family study. Arch Gen Psychiatry 1998;55(6):492-499.

82. Kendler KS. Genetic epidemiology in psychiatry. Arch Gen Psychiatry 1995;52(5):895-899.

83. Beaty TH, Khoury MI. Interface of genetics and epidemiology. Epidemiol Rev 2000;22(1):120-125.

84. Eaton W W. Studying the natural history of psychopathology. En: Tsuang MT,Tohen M, G wendolyn E.P. Zahner GEP, ed.Textbook in psychiatric epidemiology. N ueva York (N Y). John W iley-Liss; 1995. 85. Robins LN . Psychiatric epidemiology- A historic review. Soc Psychiatry Psychiatr Epidemiol 1990;25(1): 16-26.

86. D ohrenwend BP, Levav I, Shrout PE, Schwartz S, N aveh G, Link BG et al. Socioeconomic status and psychiatric disorders:The causationselection issue. Science 1992:255(5047):946-952.

87. A braido-Lanza AF, D ohrenwend BP, N g-Mak DS,Tumer JB.The Latino mortality paradox:A test of the «salmon bias» and healthy migrant hypotheses. Am J Public Health 1999; 89(10): 1543-1548.

88. Johnson JG, Cohen P, D ohrenwend BP, Link BG, Brook JS. A longitudinal investigation of social causation and social selection processes involved in the association between socioeconomic status and psychiatric disorders. J Abnorm Child Psychol 1999; 8 (3):490-499. 89. Kessler RC. Psychiatric epidemiology: Selected recent advances and future directions. Bull W orld Health 0 rgan 2000;78(4):464-474. 90. Dohrenwend BP.A psychosocial perspective on the past and future of psychiatriv epidemiology. Am J Epidemiol 1998; 147 (3): 222-231. 91.Anthony JC, Eaton W W, Henderson AS. Looking to the future in psychiatric epidemiology. Epidemiol Rev 1995;17(1):240-242.

92. Mann A.The evolving face of psychiatric epidemiology. $\mathrm{Br}$ J Psychiatry 1997: 171:314-318.

93. Merikangas KR. Editorial.The next decade of psychiatric epidemiology. Int J Methods Psychiatric Res 1999;8:1-5.

94. Eaton W W, Merikangas KR. Psychiatric epidemiology: Progress and prospects in the year 2000. Epidemiol Rev 2000;22 (1):29-34. 95.Tamez-G onzález S, O rtiz-Hernández L, Martínez-Alcántara S, Méndez-Ramírez I. Riesgos y daños a la salud derivados del uso de videoterminal. Salud Publica Mex 2003;45:171-180.

96. García-Silverman S. Un modelo explicativo de la conducta hacia la enfermedad mental. Salud Publica Mex 2002;44:289-296.

97. Salgado de Snyder VN , Díaz-Pérez MJ, González-Vázquez T. Modelo de integración de recursos para la atención de la salud mental en la población rural en México. Salud Publica Mex 2003;45:19-26. 98. C araveo-A nduaga JJ, Colmenares-Bermúdez E, Martínez-Vélez N A. Síntomas, percepción y demanda de atención en salud mental en niños y adolescentes de la ciudad de México. Salud Publica Mex 2002;44: 492-498.

99. Henderson S. Epidemiology of mental disorders:The current agenda. Epidemiol Rev 2000;22(1):24-28.

100. Séquier A, Sto ebner A, G ourgou S. Lence JJ, Bonifaci C, SanchoGarnier H. Métodos educativos en la prevención del tabaquismo, en escolares del Departamento del Herault, Francia. Salud Publica Mex 2002;44(S1):S93-S100. 